January 1993

\title{
DECOHERENCE FUNCTIONAL
}

\section{AND \\ INHOMOGENEITIES IN THE EARLY UNIVERSE}

\author{
${ }^{1}$ R. Laflamme ${ }^{*} \&{ }^{2}$ A. Matacz ${ }^{* *}$ \\ ${ }^{1}$ Theoretical Astrophysics, T-6, MSB288, \\ Los Alamos National Laboratory, \\ Los Alamos, NM87545, \\ U.S.A. \\ 1 Department of Physics, \\ University of Adelaide, \\ PO Box 498 Adelaide, \\ Australia 5001.
}

\begin{abstract}
We investigate the quantum to classical transition of small inhomogeneous fluctuations in the early Universe using the decoherence functional of Gell-Mann and Hartle. We study two types of coarse graining; one due to coarse graining the value of the scalar field and the other due to summing over an environment. We compare the results with a previous study using an environment and the off-diagonal rule proposed by Zurek. We show that the two methods give different results.
\end{abstract}

\footnotetext{
* Email: laf@tdo-serv.lanl.gov

** Email: amatacz@physics.adelaide.edu.au
} 


\section{1) Introduction.}

The gravitational instability picture for galaxy formation assumes that the early Universe started with a very smooth background on which small density fluctuations were superimposed. It is these small fluctuations which are ultimately responsible for the structure in the present Universe. They have been amplified by the gravitational interaction since the beginning of the matter dominated era and produced the galaxies we see.

In the sixties and seventies no theories were able to predict the existence of these perturbations, they were just postulated to be there. Zeldovich ${ }^{1}$ and Harrisson ${ }^{2}$ suggested that in order to fit the observation the initial spectrum of these perturbations must be roughly scale free. In $1980 \mathrm{Guth}^{3}$ proposed the Inflationary scenario to solve the horizon, flatness and monopole problems of the Big Bang. This scenario asserts that the Univserse went through a phase of very rapid expansion in its very early stage. The Universe would have expanded by a factor of at least $10^{28}$ in a mere $10^{-32}$ seconds.

It was soon realised that this very rapid expansion would have very interesting effect on fields especially the inhomogeneous part of the inflaton ${ }^{4,5}$. The state of small inhomogeneities undergoes parametric amplification during the inflationary period as soon as as a given mode crosses the Hubble radius. This lead to a scale free spectrum. It was therefore suggested that these inhomogeneities gave rise to the needed density fluctuations in the early Universe.

It was argued that the quantum expectation value of the square of the field $<\phi^{2}>$

can be interpreted as a statistical average of classical perturbations. The argument used by Guth \& $\mathrm{Pi}^{2}$ was that $\left(<\phi^{2}><\pi_{\phi}^{2}>\right)^{1 / 2}>>\hbar$ and thus quantum mechanical affects should be negligible.

Interestingly enough this consideration is not invariant under linear canonical transformations. To see this more clearly the Wigner function can be calculated. In general the Wigner function is not positive and cannot represent a classical phase space density distribution but in the case of a gaussian state it is positive. So let's assume it can give us an idea of the classical phase space distribution. The Wigner function is defined as

$$
f_{w}\left(\phi, \pi_{\phi}\right)=\frac{1}{2 \pi} \int d \Delta e^{i 2 \pi \pi_{\phi} \Delta} \rho(\phi-\Delta, \phi+\Delta)
$$


where $\rho$ is the state of the system. Figure 1 depicts the $1-\sigma$ contour of the Wigner function for a mode $\mathrm{k}$ of a massless scalar field in the Bunch-Davies vacuum. Initially it is an ellipse rotating with frequency $k / 2 \pi$ whose amplitude is adiabatic. As soon as the wavelength of the mode crosses the Hubble radius the ellipse stop rotating and gets elongated in the momentum direction. As seen from the picture the variance of $\phi$ and $\pi_{\phi}$ are such that $\left(<\phi^{2}><\pi_{\phi}^{2}>\right)^{1 / 2}>>\hbar$ but the surface of this ellipse remains $\hbar$. Using a linear canonical transformation so that $\tilde{\phi}$ and $\tilde{\pi}_{\phi}$ are in the direction of the proper axis of the ellipse would give $\left(<\tilde{\phi}^{2}><\tilde{\pi}_{\phi}^{2}>\right)^{1 / 2}=\hbar$. It is therefore difficult to understand why the quantum mechanical average can be substituted by a statistical one.

Figure 1. $1-\sigma$ contour of a Wigner function for a mode of a scalar field which has crossed the Hubble radius. The area remains $\hbar$ even if $\left(<\phi^{2}><\pi_{\phi}^{2}>\right)^{1 / 2}>>\hbar$.

A lot of effort has recently been focused on understanding the transition between quantum and classical mechanics. It has been proposed that a measure of the classicality of a system is obtained by investigating the off-diagonal terms of the density matrix ${ }^{6,7}$.

In ref [8] such a criteria was used to investigate the classicality of the inhomogeneous quantum fluctuations in the inflationary period. It was shown that these fluctuations were 
not classical if they were not interacting with an environment. A simple model of an environment represented by a single scalar field was constructed and it was shown that the off diagonal terms of the density matrix, in the configuration space basis, decreased rapidly as soon as the mode left the Hubble radius. The main problem with this approach is the assumption that when the off-diagonal terms in configuration space vanish, the system behaves classically. The density matrix gives information about the field at a given instant in time but it does not indicate how a small cell in phase space evolves. It tells us only how the sum of all these cells evolve. Classical behaviour requires each small cell of phase space to evolve independently of the others, that is, for there to be no quantum interference between different cells of phase space.

In this paper we want to investigate a different approach to classicality, the one using the decoherence functional ${ }^{9,10}$. After introducing the method in section 2 we investigate its consequences for perturbations in the inflationary universe. We study two types of coarse graining; one due to coarse graining of the value of the scalar field and the other by summing over an environment as in ref [8]. We compare the coherence length of the decoherence functional coarse grained from an environment with that of the density matrix and find striking differences. We discuss and conclude in section 3.

\section{2) The Decoherence Functional.}

In recent years an approach to the quantum to classical transition has considered not the state or eigenvalues of operators at a given time but rather focused on histories defined by a series of the value of fields at a different time ${ }^{9,10,11,12}$. The idea is that a necessary condition for a system to be thought of as classical is that the probability sum rule for different histories should be obeyed. In other words interference should vanish. In such a case Griffith called them consistent histories.

The tool to calculate the probability of an history is the decoherence functional. The fine grained decoherence functional for histories defined by the positions at all times can be defined through the path integral

$$
D\left[h^{\prime}, h\right]=\delta\left(q_{f}^{\prime}-q_{f}\right) \exp i\left[S\left[q^{\prime}(\eta)\right]-S[q(\eta)]\right] \rho\left(q_{i}^{\prime}, q_{i}, \eta_{i}\right)
$$

where $S$ is the action for the given history. In order for two histories $h^{\prime}, h$ to be consistent 
the decoherence functional must be diagonal. Except for very special cases, fine grained histories will not decohere. A possible way to get consistent histories is to coarse grain them.

A coarse graining of this decoherence functional can be obtained by looking at histories with approximate position or momenta, or by summing over some field which is considered an environment. It is the latter case which corresponds to the the decoherence studied by Zeh $^{7}$ and Zurek ${ }^{8}$. A coarse graining can be defined as

$$
D^{c}\left[h^{\prime}, h\right]=\int_{h^{\prime}} \mathcal{D} q^{\prime} \int_{h} \mathcal{D} q \delta\left(q_{f}^{\prime}-q_{f}\right) \exp i\left[S\left[q^{\prime}(\eta)\right]-S[q(\eta)]\right] \rho\left(q_{i}^{\prime}, q_{i}, \eta_{i}\right)
$$

The path integral over $q(\eta)$ is over all paths that start at $q_{i}$ at $\eta_{i}$, pass through the intervals $\Delta^{1}\left(\eta_{1}\right), \Delta^{2}\left(\eta_{2}\right) \ldots, \Delta^{n}\left(\eta_{n}\right)$ at $\eta_{1}, \eta_{2} \ldots \eta_{n}$ and wind up at $q_{f}$ at time $\eta_{f}$. Similarly for $q^{\prime}(\eta)$ which goes through primed interval but end at the same endpoint $q_{f}$.

We will evaluate (2.2) for a scalar field evolving in the early universe both for coarse graining of the field or of an environment. A crucial question is how to model this environment. Any realistic model will be very complicated and hard to analyze. However, the basic physics should emerge from the simplest models. Hence we use a model which can be solved exactly: the system is a real massless scalar field $\Phi_{1}$, (the inflaton), the environment is taken to be a second massless real scalar field $\Phi_{2}$ interacting with $\Phi_{1}$ by their gradients. This will permit us to compare our results with the ones in ref.[8]. We consider the fields in the de Sitter phase of an expanding Universe with scale factor $a(t)=\exp (H t)$, where $H$ is the Hubble constant.

The action of system and environment is

$$
I=\int d^{4} x \sqrt{g} \frac{1}{2}\left(\left(\partial_{\mu} \Phi_{1}\right)^{2}+\left(\partial_{\mu} \Phi_{2}\right)^{2}+2 c\left(\partial_{\mu} \Phi_{1} \partial^{\mu} \Phi_{2}\right)\right)
$$

where $g$ is the determinant of the background metric with line element given by

$$
d s^{2}=a^{2}\left(-d \eta^{2}+d x_{i}^{2}\right)
$$

$c$ is a constant measuring the strength of interaction between system and environment. We shall normalize the conformal time $\eta$ such that $\eta$ ranges between $-\infty$ and 0 and $a=-(H \eta)^{-1}$ with $H^{-1}$ being the Hubble radius. 
We will study two types of coarse graining. The first one will consist in summing over the field $\Phi_{2}$ which mimicks the environment. The second one will consist of coarse graining the value of the field $\Phi_{1}$.

Our Lagrangian is quadratic in the derivatives of the fields and can hence be diagonalized using fields $\Phi_{+}$and $\Phi_{-}$for which the interaction term disappears. The coherences in the quantum state between $\Phi_{+}$and $\Phi_{-}$are only given by the initial conditions. For example, we could choose an initial state where these coherences vanish. In this case, a pure state gives rise to a pure state reduced density matrix when summing over one of the fields. Decoherence of one field cannot occur by summing over the other one. We, however, suppose that the inflaton and the environment do not form the diagonal basis. This assumption is reasonable since any inflaton field (whose reduced density matrix we want) will interact with gravitational perturbations (part of the environment).

We can expand the fields in harmonics in a box of fixed comoving volume (physical volume $\left.a^{3}\right)$ and investigate a particular wavenumber $k=\left(k_{x}^{2}+k_{y}^{2}+k_{z}^{2}\right)^{1 / 2}$. As there is no coupling between modes with different $k$, we can consider a single wavelength and drop the index $k$ for convenience. The Lagrangian reduces to

$$
L(q, \dot{q}, r, \dot{r}, \eta)=\frac{a^{2}(\eta)}{2}\left[\dot{q}^{2}+\dot{r}^{2}-k^{2} q^{2}-k^{2} r^{2}+2 c\left(\dot{q} \dot{r}-k^{2} q r\right)\right]
$$

For simplicity we will consider histories described by only two values of $q$ 's, the value at time $\eta_{i}$ and $\eta_{f}$. When a hamiltonian exists we ca $\mathrm{n}$ rewrite the decoherence functional in the operator formalism as

$$
D\left(h_{1}, h_{2}\right)=\operatorname{Tr}\left[U\left(\eta_{f}-\eta_{i}\right) P_{q_{1} r_{1}}^{\sigma} \rho\left(\eta_{i}\right) P_{q_{2} r_{2}}^{\sigma} U^{\dagger}\left(\eta_{f}-\eta_{i}\right) P_{q_{f} r_{f}}^{\sigma}\right]
$$

In order to compare with the results using the density matrix, we will look for histories where $r$ is considered as an environment and at first $q$ is fine grained. The two histories that we will consider are the define by starting at either $q_{1}$ or $q_{2}$ at $\eta_{i}$ and ending up at $q_{f}$. The decoherence functional becomes

$D\left(h_{1}, h_{2}\right)=N \int d r_{1} d r_{2} d r_{f} K^{*}\left(q_{f}, r_{f}, \eta_{f} ; q_{2}, r_{2}, \eta_{i}\right) K\left(q_{f}, r_{f}, \eta_{f} ; q_{1}, r_{1}, \eta_{i}\right) \rho\left(q_{1}, r_{1} ; q_{2}, r_{2}, \eta_{i}\right)$ 
and the propagator $K$ is

$$
\begin{aligned}
K\left(q_{f}, r_{f}, \eta_{f} ; q_{1}, r_{1}, \eta_{i}\right)=\left[\frac{i k^{3}}{2 \pi H^{2} x}\right] & \exp \frac{i}{2 x}\left[\frac{\left(q_{f}^{2}+r_{f}^{2}+2 c q_{f} r_{f}\right) y_{f}}{H^{2} \eta_{f}^{2}}+\frac{\left(q_{1}^{2}+r_{1}^{2}+2 c q_{1} r_{1}\right) y_{i}}{H^{2} \eta_{i}}\right. \\
& \left.+\frac{2 k^{3}\left(q_{f} q_{1}+r_{f} r_{1}+c q_{f} r_{1}+c r_{f} q_{1}\right)}{H^{2}}\right]
\end{aligned}
$$

where

$$
\begin{gathered}
x=-k^{2} \eta_{f} \eta_{i} \sin k \Delta+k \Delta \cos k \Delta-\sin k \Delta \\
y_{f}=-k^{3} \eta_{f} \eta_{i} \cos k \Delta-k^{2} \eta_{f} \sin k \Delta \\
y_{i}=-k^{3} \eta_{f} \eta_{i} \cos k \Delta+k^{2} \eta_{i} \sin k \Delta
\end{gathered}
$$

and $\Delta=\eta_{f}-\eta_{i}$. If we assume that the initial to be

$$
\psi\left(q, r, \eta_{i}\right)=a \exp -b\left[q^{2}+r^{2}+2 \alpha q r\right]
$$

where $b$ is complex and $\alpha$ is real, we find that

$$
D\left(h_{1}, h_{2}\right)=D \exp \frac{i\left(1-c^{2}\right)}{2 x}\left[\frac{y_{i}\left(q_{1}^{2}-q_{2}^{2}\right)}{H^{2} \eta_{i}^{2}}+\frac{2 k^{3} q_{f}\left(q_{1}-q_{2}\right)}{H^{2}}\right] \exp -\left(q_{1}^{2} A+q_{2}^{2} A^{*}+q_{1} q_{2} B\right)
$$

where

$$
\begin{aligned}
& A=\left[b^{2}\left(1-\alpha^{2}\right)+b b^{*}\left(1+c^{2}-2 c \alpha\right)\right] /\left(b+b^{*}\right) \\
& B=-2 b b^{*}(c-\alpha)^{2} /\left(b+b^{*}\right) \\
& D=a a^{*}\left(\frac{\pi}{b+b *}\right)^{1 / 2} \frac{k^{3}}{2 \pi H^{2} x} .
\end{aligned}
$$

This decoherence functional predicts a certain coherence length $L_{d f}$, which is the maximum length (in configuration space squared) between histories over which interference is not exponentially suppressed. However to get a better measure of the decoherence of the decoherence functional, $D_{d f}$, we should divide $L_{d f}$ by the probability width of the system 
$P_{d f}$. This is obtained by setting $q_{1}=q_{2}$ in (2.11) and finding the length in configuration space squared where the probability not exponentially suppressed. We find that

$$
D_{d f}=\frac{P_{d f}}{L_{d f}}=\frac{A+A^{*}-B}{A+A^{*}+B}=1+\frac{4 b b^{*}(c-\alpha)^{2}}{\left(1-\alpha^{2}\right)\left(b+b^{*}\right)^{2}}
$$

When $D_{d f}>1$ we have significant histories decoherence. This measure for histories decoherence can be compared to the one used in ref[8], using the off-diagonal terms of the reduced density matrix which is given by

$\rho_{\text {red }}\left(q_{1}, q_{2}, \eta_{i}\right)=\int \psi\left(q_{1}, r, \eta_{i}\right) \psi^{*}\left(q_{2}, r, \eta_{i}\right) d r=a a^{*}\left(\frac{\pi}{b+b^{*}}\right)^{1 / 2} \exp -\left(q_{1}^{2} f+q_{2}^{2} f^{*}+q_{1} q_{2} g\right)$

where

$$
g=-2 \alpha^{2} b b^{*} /\left(b+b^{*}\right), \quad f=\left(b^{2}\left(1-\alpha^{2}\right)+b b^{*}\right) /\left(b+b^{*}\right) .
$$

In this case an analogous measure used was

$$
D_{d m}=\frac{P_{d m}}{L_{d m}}=\frac{f+f^{*}-g}{f+f^{*}+g}=\frac{(b+b)^{2}-\alpha^{2}\left(b-b^{*}\right)^{2}}{\left(1-\alpha^{2}\right)\left(b+b^{*}\right)^{2}} .
$$

This expression was analysed in ref[8] for the Bunch-Davies initial condition which corresponds to

$$
\alpha=c, \quad b=\frac{k^{2}}{2 H^{2} \eta_{i}\left(k \eta_{i}+i\right)} .
$$

The limit of interest is long after Hubble crossing where $\left|k \eta_{i}\right|<<1$ which implies that $b \approx k^{3} /\left(2 H^{2}\right)-i k^{2} /\left(2 H^{2} \eta_{i}\right)$. In this limit we see that $D_{d m}>>1$, however $D_{d f}=1$. Thus we have a situation where an arbitrarily large decoherence of the configuration space density matrix corresponds to a maximally coherent decoherence functional. The BunchDavies vacuum is the ground state. If we perturb the initial coupling away from $c$ then we will have some histories decoherence as well as density matrix decoherence. In this case the propagator will generate an imaginary contribution to $\alpha$. This imaginary part will modify (2.13) and (2.16) in a way that cancels the divergence which would otherwise occur for $\alpha \neq c(\alpha$ real $)$ in the limit $k \eta_{i} \rightarrow 0$. In this case there may be closer relationship 
between the two decoherence measures. We can get an idea of the relative strengths of the two decoherence measures (for real $\alpha$ ) by taking their ratios. We find

$$
\frac{L_{d m}}{L_{d f}}=\frac{A+A^{*}-B}{f+f^{*}-g}=1+\frac{c(c-2 \alpha) b b^{*}}{(\text { re } b)^{2}+\alpha^{2}(i m b)^{2}} .
$$

We can see that if $c=0$ the two coherence lengths agree. This can be easily seen form (2.7). The integral over $r_{f}$ will be proportional to $\delta\left(r_{1}-r_{2}\right)$ and thus the decoherence functional is proportional to the initial density matrix. It is rather surprising however that turning on the interaction from $c=0$ to $c=2 \alpha$ will increase the coherence of the decoherence functional relative to the density matrix. However for $c>2 \alpha$ the coherence length of the density matrix is larger than the coherence length of the decoherence functional. This shows that in this case there is no obvious correlation between the two decoherence measures and that the decoherence of the density matrix does not imply the decoherence of the decoherence functional or vice-versa.

Another possibility to obtain decoherence is to coarse grain our system in configuration space, to which we turn now. In this case the histories are not defined by precise values of $q$ but by a range determined by $\sigma$ (a variance) around a given value. The decoherence functional can then be obtained by integrating the fine grained one. The $P$ s are projectors on a range $2 \sigma$ of the fields. They are rather tedious to work with analytically. It will be useful to keep the analytical result simple so we use the gaussian pseudo-projectors

$$
P_{q_{i} r_{i}}^{\sigma}=\frac{1}{\pi^{1 / 2} \sigma} \int_{-\infty}^{\infty} d z_{i} d r_{i} \exp \left(\frac{-\left(z_{i}-q_{i}\right)^{2}}{\sigma^{2}}\right)\left|z_{i}, r_{i}\right\rangle\left\langle r_{i}, z_{i}\right|
$$

They are not exactly projectors as

$$
P^{2} \neq P
$$

but are a sufficiently good approximation for our purpose. Substituting (2.19) into (2.6) we get

$$
D^{c}\left(h_{1}, h_{2}\right)=\int d z_{1} d z_{2} d z_{3} \exp \left[-\frac{\left(z_{1}-q_{1}\right)^{2}}{\sigma^{2}}-\frac{\left(z_{2}-q_{2}\right)^{2}}{\sigma^{2}}-\frac{\left(z_{3}-q_{f}\right)^{2}}{\sigma^{2}}\right] D\left(h_{1}, h_{2}\right)
$$


where $D\left(h_{1}, h_{2}\right)$ is given by (2.11). We choose the Bunch-Davies initial condition (2.17) for (2.11). This is the most natural initial state to choose, it considerably simplifies the algebra and it ensures by virtue of (2.13) that any decoherence obtained will not be due to the environmental coarse grain. We can rewrite the result in terms of $Q=q_{1}+q_{2}$ and $\delta=q_{1}-q_{2}$ and get

$$
D^{c}\left(h_{1}, h_{2}\right)=N \exp \left[a_{1} Q^{2}+a_{2} \delta^{2}+a_{3} q_{f}^{2}+a_{4} Q \delta+a_{5} q_{f} \delta+a_{6} Q q_{f}\right]
$$

where

$$
\begin{aligned}
& a_{1}=\frac{-1}{2 \sigma^{2}}+\frac{1}{4 \sigma^{4}} \frac{M+M^{*}+2 V}{M M^{*}-V^{2}} \\
& a_{2}=\frac{-1}{2 \sigma^{2}}+\frac{1}{4 \sigma^{4}} \frac{M+M^{*}-2 V}{M M^{*}-V^{2}} \\
& a_{3}=-\frac{V\left(M+M^{*}-2 V\right)}{\sigma^{2}\left(M M^{*}-V^{2}\right)} \\
& a_{4}=\frac{M-M^{*}}{2 \sigma^{4}\left(M M^{*}-V^{2}\right)} \\
& a_{5}=\frac{i V^{1 / 2}\left(M+M^{*}-2 V\right)}{\sigma^{3}\left(M M^{*}-V^{2}\right)} \\
& a_{6}=\frac{i V^{1 / 2}\left(M-M^{*}\right)}{\sigma^{3}\left(M M^{*}-V^{2}\right)}
\end{aligned}
$$

with

$$
\begin{aligned}
& M=\frac{1}{\sigma^{2}}+\left(1-c^{2}\right) b^{*}+\frac{i\left(1-c^{2}\right) y_{i}}{2 x H^{2} \eta_{i}^{2}}+\frac{\left(1-c^{2}\right)^{2} k^{6} \sigma^{2}}{4 x^{2} H^{4}} \\
& V=\frac{\left(1-c^{2}\right)^{2} k^{6} \sigma^{2}}{4 x} .
\end{aligned}
$$

Investigating (2.22-24) shows that coarse grained histories that are determined by their approximate positions at various times are not exactly consistent. Exact decoherence is rather difficult to obtain so we investigate approximate decoherence. Histories are approximatively consistent if

$$
\left|\operatorname{Re} D\left(h_{1}, h_{2}\right)\right|<\varepsilon M i n\left[\operatorname{Re} D\left(h_{1}, h_{1}\right), \operatorname{Re} D\left(h_{2}, h_{2}\right)\right]
$$


This only means that the off-diagonal are much smaller than its corresponding diagonal part and thus the classical sum rules applies approximatively. $\varepsilon$ controls how good the approximation is. If we consider symmetrical histories $\left(q_{1}=-q_{2}, q_{f}=0\right)$ then it is easy to see from (2.22) that (2.25) translates mathematically as

$$
\frac{a_{1}}{a_{2}}<<1
$$

We also want to be able to interpret the quantum mechanical average of operators as a statistical one. This implies that the coarse grain should be smaller than the fluctuations $(\Delta q)^{2}$ of the field. For the Bunch Davies vacuum (2.17), in the long after Hubble crossing limit, $(\Delta q)^{2} \rightarrow H^{2} / k^{3}$ hence we require

$$
\sigma^{2}<<\frac{H^{2}}{k^{3}}
$$

In general (2.23) will be very long expressions. However they simplify greatly in the late time limit which implies that from $(2.9 \mathrm{a}, 2.9 \mathrm{c}) y_{i} \rightarrow-k^{3} \eta_{i}^{2}$ and $x \rightarrow \frac{-k^{3} \Delta}{3}\left(3 \eta_{i} \eta_{f}+\Delta^{2}\right)$. We further consider the limit $\Delta \rightarrow 0, \eta_{i} \rightarrow 0$ and $\eta_{f} \rightarrow 0$. We can take this limit while keeping an arbitrary constant proper time interval, $\delta t$ since $\frac{d \eta}{d t}=-H \eta$. In this limit we find that

$$
\begin{aligned}
& a_{1} \rightarrow \frac{-1}{2 \sigma^{2}}+\frac{1}{\sigma^{4}}\left[\frac{3}{\sigma^{2}}+\frac{\left(1-c^{2}\right) k^{3}}{H^{2}}\right]^{-1} \\
& a_{2} \rightarrow \frac{-1}{2 \sigma^{2}} \\
& a_{3} \rightarrow \frac{-\left(1-c^{2}\right)}{\sigma^{2}}\left[\frac{2 H^{2}+\left(1-c^{2}\right) k^{3} \sigma^{2}}{3 H^{2}+\left(1-c^{2}\right) k^{3} \sigma^{2}}\right] \\
& a_{4} \rightarrow a_{5} \rightarrow 0 \\
& a_{6} \rightarrow \frac{2\left(1-c^{2}\right)}{\sigma^{4}}\left[\frac{3}{\sigma^{2}}+\frac{\left(1-c^{2}\right) k^{3}}{H^{2}}\right]^{-1} .
\end{aligned}
$$

For our model in the late time limit (2.26) becomes

$$
\frac{a_{1}}{a_{2}} \approx 1 / 3
$$


Equation (2.29) tells us that there is weak decoherence but not a significant amount. To get a better feel for this number it is worth comparing it to the long before Hubble crossing limit which gives $a_{1} / a_{2}=1$. Thus the after Hubble crossing limit does lead to some decoherence but not a significant amount.

Assuming decoherent histories $(\delta=0, q=Q / 2)$ we find that (2.22) becomes, using $(2.28)$ and $(2.27)$

$$
D^{c}(h, h) \approx N \exp \left[\frac{-2}{3 \sigma^{2}}\left(q-q_{f}\right)^{2}\right]
$$

Thus at late times the histories would be peaked about $q_{f} \approx q$ which is exactly the behavior of the classical motion.

\section{3) Discussion and conclusion.}

We see from (2.16) that the decoherence in the density matrix is due purely to the phase of the wave-function. This dependence on the phase is interesting since the phase can always be changed by a point transformation on the Lagrangian. We can see this as follows. A point transformation will transform the Lagrangian as

$$
L(\vec{q}(t), \dot{\vec{q}}(t)) \rightarrow L(\vec{q}(t), \dot{\vec{q}}(t))-\frac{d}{d t} f(\vec{q}(t), t)
$$

which in turn means that the action transforms as

$$
S[q(t)] \rightarrow S[q(t)]-f\left(\vec{q}_{f}, t_{f}\right)+f\left(\vec{q}_{i}, t_{i}\right)
$$

This point transformation doesn't affect the classical equation of motion because they are derived from the stationary action condition $\delta S=S[q(t)]-S[q(t)+\delta q(t)]=0$ where $\delta q(t)$ vanishes at the endpoints. However from the general expression $U\left(\vec{q}_{f}, t_{f} ; \vec{q}_{i}, t_{i}\right)=$

$N \sum_{\text {paths }} e^{i S}$ for the quantum propagator we can see that under the transformation (3.2) the quantum propagator transforms as

$$
U\left(\vec{q}_{f}, t_{f} ; \vec{q}_{i}, t_{i}\right) \rightarrow e^{-i f\left(\vec{q}_{f}, t_{f}\right)} U\left(\vec{q}_{f}, t_{f} ; \vec{q}_{i}, t_{i}\right) e^{i f\left(\vec{q}_{i}, t_{i}\right)}
$$

which in turn means that the wave function transforms as

$$
\psi(\vec{q}, t) \rightarrow e^{-i f(\vec{q}, t)} \psi(\vec{q}, t)
$$


Physics is generally considered invariant under the point transformation (3.1) because expectation values of functions of $q$ and the physical momenta $\dot{q}$ are invariant (it is important to remember that the canonical momenta does change with (3.2)). However the reduced density matrix of a subsystem in is not invariant to these point transformations. A point transformation is exactly what is being done when surface terms are dropped in a lagrangian. Using (3.3) and (3.2) we can see that the decoherence functional (2.7) is invariant under point transformations. This is an important difference between the two formalisms.

In models with more general couplings we should expect decoherence of the reduced density matrix to depend not only on the phase but also on the real part of the exponent of the wave function. In this case there might be a simpler relation between the decoherence functional and the evolution of the density matrix.

It is also interesting to investigate the influence functional for this model. Naively we might relate a diagonal decoherence functional with the existence of a noise kernel in the influence functional. Consider (2.2) where $q \rightarrow(q, r), q^{\prime} \rightarrow\left(q^{\prime}, r^{\prime}\right)$ and the $r, r^{\prime}$ coordinates are completely coarse-grained out. In this case (2.2) becomes

$D\left[h^{\prime}, h\right]=\int_{h^{\prime}} \mathcal{D} q \int_{h} \mathcal{D} q^{\prime} \int d q_{i} d q_{i}^{\prime} d q_{f} d q_{f}^{\prime} \delta\left(q_{f}-q_{f}^{\prime}\right) \exp i\left[S_{f}[q(\eta)]-S_{f}\left[q^{\prime}(\eta)\right]\right] F\left[q(\eta), q^{\prime}(\eta)\right]$

where $F\left[q(\eta), q^{\prime}(\eta)\right]$ the influence functional is

$$
\begin{aligned}
& F\left[q(\eta), q^{\prime}(\eta)\right]=\int d r_{i} d r_{i}^{\prime} d r_{f} d r_{f}^{\prime} \delta\left(r_{f}-r_{f}^{\prime}\right) \rho\left(q_{i}, r_{i} ; q_{i}^{\prime}, r_{i}^{\prime}, \eta_{i}\right) \\
& \times \int_{\left(r_{i}, r_{i}^{\prime}, \eta_{i}\right)}^{\left(r_{f}, r_{f}^{\prime}, \eta_{f}\right)} \mathcal{D} r \mathcal{D} r^{\prime} \exp i\left[S_{f}[r(\eta)]+S_{i}[q(\eta), r(\eta)]-S_{f}\left[r^{\prime}(\eta)\right]-S_{i}\left[q^{\prime}(\eta), r^{\prime}(\eta)\right]\right] .
\end{aligned}
$$

For our model (2.5) with the Bunch-Davies initial condition (2.17) we find that the influence functional is

$$
\begin{aligned}
F\left[q(\eta), q^{\prime}(\eta)\right] & =\exp \left[\frac{i c^{2}}{2} \int_{\eta_{i}}^{\eta_{f}} a^{2}\left(\dot{q}^{\prime 2}-k^{2} q^{\prime 2}\right)-\frac{i c^{2}}{2} \int_{\eta_{i}}^{\eta_{f}} a^{2}\left(\dot{q}^{2}-k^{2} q^{2}\right)\right] \\
& \times \exp \left[-\left(1-c^{2}\right) b_{i} q_{i}^{2}-\left(1-c^{2}\right) b_{i}^{*} q_{i}^{\prime 2}-\frac{c^{2} b_{f} b_{f}^{*}\left(q_{f}-q_{f}^{\prime}\right)^{2}}{b_{f}+b_{f}^{*}}\right] .
\end{aligned}
$$


A striking feature of (3.7) is the absence of a noise kernel that is typically associated with decoherence. This is due to the very special form of interaction we have chosen. The result is that in (3.5), there is no exponential suppression of widely separated histories and hence no histories decoherence. The influence functional will still not have a noise kernel even if the initial state does not have $c=\alpha$. This shows that the absence of noise kernel does not imply a coherent evolution.

We have shown that the decoherence functional shows some decoherence for the interaction given in eq.(2.3) for a wide selection of initial states. We have also shown that there is a surprising result for the case $c=\alpha$ as we have already mentioned. This case surely needs further study in order to understand why a mixed state can lead to a maximally coherent (factorizable) decoherence functional. We also considered the possibility of decoherence after Hubble crossing though coarse graining the system field. We found this led to weak decoherence after Hubble crossing but probably not enough for an effective quantum to classical transition. 


\section{References.}

1. Ya.B. Zeldovich, Mon.Not.R.Astron.Soc. 160, 1 (1972).

2. E.R. Harrisson, Phys.Rev.D. 1, 2726 (1970).

3. A. Starobinsky, Phys.Lett. 91B, 99 (1980). A. Guth, Phys.Rev. D23, 347 (1981); K.Sato, Mon.Not.R.Astron.Soc. 195 , 467 (1981); A.D. Linde, Phys.Rev.Lett. 108B, 389 (1982); A. Albrecht \& P.J. Steinhardt, Phys.Rev.Let. 48, 1220 (1980).

4. S.W.Hawking, Phys.Lett 115B,295 (1982).

5. A.H.Guth \& S.Y.Pi, Phys.Rev.Lett. 49, 1110 (1982).

6. W.Zurek, Phys.Rev. D24, 1516 (1981).

7. E.Joos \& H. Zeh, Z.Phys. B59, 223 (1985).

8. R.Brandenberger, R.Laflamme \& M.Mijić, Mod.Phys.Lett. A5, 2311 (1990).

9. J.B.Hartle, in Proceedings of the 1992 Les Houches Summer School on Gravitation and Quantizations, ed. by B. Julia, North Holland, Amsterdam and references therein.

10. M.Gell-Mann \& J.B.Hartle, Classical Equations for Quantum Systems, (to appear in Phys. Rev. D.).

11. R.Griffiths, J.Stat.Phys. 36, 219 (1984).

12. R.Omnes, Rev.Mod.Phys. 64, 339 (1992). 